\title{
Diagnostic challenge and surgical management of Boerhaave's syndrome: three diagnosed cases of spontaneous esophageal rupture
}

Jiayue Wang

Jinhua Hospital of Zhejiang University: Jinhua Municipal Central Hospital

Degang Wang

Chaoyang Central Hospital

Jianjiao Chen ( $\nabla$ cjj13390530038@126.com )

Jinhua Hospital of Zhejiang University: Jinhua Municipal Central Hospital https://orcid.org/00000002-8735-9512

\section{Case report}

Keywords: Boerhaave's Syndrome, esophageal perforation, CT, surgical management

Posted Date: September 9th, 2020

DOI: https://doi.org/10.21203/rs.3.rs-69336/v1

License: (c) (i) This work is licensed under a Creative Commons Attribution 4.0 International License.

Read Full License 


\section{Abstract}

BACKGROUND: Boerhaave's syndrome is the spontaneous rupture of the esophagus, caused by an increase of intraluminal pressure that is produced in the context of negative intrathoracic pressure. It has a high index of morbimortality, which is why it requires early diagnosis and treatment. Symptoms may vary, and diagnosis can be challenging.

CASE PRESENTATION: Case one: A 54-year-old man presented to us with sudden-onset epigastric pain radiating to the back following hematemes. His previous medical history included gastric ulcer. His physical signs suggested early shock. Combined with his medical history and physical signs, emergency doctor suspected a diagnosis of peptic ulcer with hematemesis, and esophagegastroscopy was performed. However, upper gastrointestinal endoscopy revealed a full-thickness rupture of the esophageal wall. The subsequent computed tomography (CT) showed frank pneumomediastinum and heterogeneous pleural effusion. He was subsequently referred to us in view of suspected Boerhaave's syndrome and clinical worsening. In view of hemodynamic instability with uncontrolled sepsis, he was planned for surgery. Esophageal perforation repair operation and jejunostomy was performed for him. The postoperative period was uneventful, and he was discharged.

Case two: A 62-year-old man was admitted to the emergency department with thoracic dull pain and chest distress that started after he had been vomiting several hours before presentation. On physical examination, he presented rough bronchovesicular breathing sound, and crepitant rales in lungs prompting subcutaneous emphysema. Chest CT scan showed pneumomediastinum and large left-sided pleural effusion. Esophagus fistula was confirmed by contrast esophagography. Therefore, spontaneous esophageal perforation was suspected. Then, we performed thoracotomy to repair the esophageal tear as well as to debride and irrigate the left pleural space. His vital signs remained stable intraoperatively, and his postoperative periods were uneventful with no leakage or stricture.

Case three: The patient was a 69-year old male presenting with a severe retrosternal and upper abdominal pain followed an episode of forceful vomiting. At admission, he was diaphoretic and in respiratory distress. Physical examination revealed extensive cervical and thoracic subcutaneous emphysema but was otherwise unremarkable. A thoracic CT scan revealed a rupture in the left distal part of the oesophagus, a pneumomediastinum and left-sided pleural effusions. Conservative treatment, with cessation of oral intake, nasogastric suction, administration of intravenous fluids and parenteral nutrition, intravenous broad-spectrum anti-biotics, proton pump inhibitors and drainage of the pleural effusion by left-sided thoracostomy, failed to improve disease conditions. Open thoracic surgery was performed with debridement and drainage of the mediastinum and the pleural cavity, after which he made a slow but full recovery.

CONCLUSIONS: We highlight that early diagnosis and appropriate surgical treatment are essential for optimum outcome in patients with esophageal rupture. We emphasize the importance of critical care support, particularly in the early stages of management. 


\section{Introduction}

Boerhaave's syndrome is a life-threatening condition characterized by a disruption of the distal esophagus due to a barotrauma that results in contamination of the mediastinum and pleural cavity with gastric contents[1]. Symptoms may vary, and diagnosis can be challenging, as the classic triad of Mackler (vomiting, lower thoracic pain and subcutaneous emphysema) is present in a few cases [2]. A significant delay between perforation and treatment often leads to mediastinitis followed by septic shock and multiorgan failure [3]. For emergency physician, what counts is chest radiology examination, almost all abnormalities are visible. Although the optimum treatment strategy has not been normalized, surgery program gets first priority among all therapeutic approaches. Here we describe three typical cases of Boerhaave's syndrome with representative clinical symptoms and imaging manifestations, we developed multimodal treatment strategies and achieved desired effect.

\section{Case One}

A 54-year-old man with a previous medical history of gastric ulcer developed sudden-onset epigastric pain radiating to the back following hematemesis and presented to the emergency department. His blood pressure was $121 / 69 \mathrm{mmHg}$, pulse rate was 110 beats/min, and percutaneous oxygen saturation $93 \%$. His axillary temperature was $37.2^{\circ} \mathrm{C}$. And his laboratory results of routine blood tests were normal by the time of presentation. Initially, emergency doctor suspected a diagnosis of peptic ulcer with hematemesis, and esophagegastroscopy was performed. However, upper gastrointestinal endoscopy revealed a fullthickness rupture of the esophageal wall, apart from this the gastroesophageal mucosa was normal and, in particular, there were no signs of ischemia (Fig. 1). Immediately, the patient underwent chest computed tomography (CT) scan which showed frank pneumomediastinum and heterogeneous pleural effusion (Fig. 2). We considered the occurrence of spontaneous esophageal rupture known as Boerhaave's syndrome. Therefore, we performed esophageal perforation repair operation and jejunostomy for him. Postoperatively, the patient was transferred to the ICU for further conservative treatment. He recovered after a 25-day hospital stay, with feeding through jejunum nutrient catheter. Four months later, we removed his jejunum nutrient catheter.

\section{Case Two}

A 62-year-old man was admitted to the emergency department with thoracic dull pain and chest distress that started after he had been vomiting several hours before presentation. At admission, his blood pressure was $116 / 78 \mathrm{mmHg}$, pulse rate was 100 beats/min, percutaneous oxygen saturation was $96 \%$ and axillary temperature was $36.5^{\circ} \mathrm{C}$. On physical examination, he presented rough bronchovesicular breathing sound, and crepitant rales in lungs prompting subcutaneous emphysema. His abdomen was smooth without tenderness or rebound pain. Laboratory results of routine blood tests and biochemical examination were normal by the time of presentation. A chest CT scan showed pneumomediastinum and large left-sided pleural effusion, and possible esophagus tear was showed in chest CT (Fig. 3).

Esophagus fistula was further confirmed by contrast esophagography (Fig. 4). Therefore, spontaneous 
esophageal perforation was suspected. As the patient's general condition was progressively deteriorating, we immediately arrange emergency surgery for him. In consideration of narrow tearing site and scope, esophageal repair under endoscopy was not suitable. Thus, we performed thoracotomy to repair the esophageal tear as well as to debride and irrigate the left pleural space. Postoperatively, the patient was transferred to the intensive care unit for further conservative treatment. A contrast esophagography findings obtained on postoperative day 12 demonstrated no sign of esophageal leak, and then he started an oral liquid diet. After an ICU stay of 20 days, the patient was discharged.

\section{Case Three}

A 69-year-old man with a history of hypertension was referred to our emergency department with a severe retrosternal and upper abdominal pain followed an episode of forceful vomiting after he drank a bottle of beer at home three hours ago. At admission, he was diaphoretic and in respiratory distress. Blood pressure was $185 / 98 \mathrm{mmHg}$, pulse rate was 105 beats/min, oxygen saturation was $94 \%$ and core temperature was $36.3^{\circ} \mathrm{C}$. Physical examination revealed extensive cervical and thoracic subcutaneous emphysema but was otherwise unremarkable. Laboratory results were normal by the time of presentation. A thoracic CT scan revealed a rupture in the left distal part of the oesophagus, a pneumomediastinum and left-sided pleural effusions (Fig. 5). Conservative treatment, with cessation of oral intake, nasogastric suction, administration of intravenous fluids and parenteral nutrition, intravenous broad-spectrum anti-biotics, proton pump inhibitors and drainage of the pleural effusion by left-sided thoracostomy, failed to improve disease conditions. Open thoracic surgery was performed with debridement and drainage of the mediastinum and the pleural cavity, after which he made a slow but full recovery.

\section{Discussion And Conclusion}

Boerhaave's syndrome is defined as spontaneous esophageal rupture due to sudden increase in esophageal pressure accompanied by inability of the cricopharyngeal sphincter relaxation[4]. It is a rare but life-threatening condition, for absence of mucous muscularity in esophagus allows food remains and gastric juices to enter into the mediastinum, resulting in extensive infection of the mediastinum and pleural cavities if without proper treatment.

Clinical manifestations at the disease's earliest stages are non-specific, depending on the site of oesophageal perforation and time eplapsed between its development and examination. Presence of Mackler triad (vomiting, lower thoracic pain and subcutaneous emphysema) always prompts high possibility of irretrievability. It is quite essential to shorten the length of time between symptom manifestation and proper surgical treatment [5]. Imaging examination is of great significance for early diagnosis of Boerhaave's syndrome. Chest radiology examination findings such as pleural effusion or pneumomediastinum are of great significance for diagnosis. Thoracic CT is helpful in differential diagnosis with other traumatic esophageal injury. Further contrast esophagography may provide exact evidence of contrast leakage from the oesophageal lumen to make a definite diagnosis. Although 
endoscopy may provide direct vision evidence, we don't recommend endoscopy as routine checking methods for its invasiveness may deteriorate the lacerated condition. Early diagnosis and management ('golden early 24-h rule') are crucial for benign outcome in patients with rupture of the oesophagus.

The therapeutic choices for Boerhaave's syndrome generally include conservative, surgical and endoscopic treatments. The appropriate management of esophageal perforation is a controversial issue. Although there is as of yet no definitive treatment approach, according to current retrospective analysis and clinical experiences, application of operative intervention to repair breakage primarily is considered as priority in current management of Boerhaave's syndrome, especially in the condition as we reported that the patient had presented signs of contamination of the pleural cavity and mediastinum[6].

Traditional thoracotomy is still practical in emergency surgery for its intuition. This surgical treatment is widely accepted as preferred treatment if tissues were viable.

It is worth noting that introduction of video-assisted thoracoscopic surgery (VATS) offers us more surgical choices for Boerhaave's syndrome. Some clinicians have progressed zealously attempt in application of VATS for Boerhaave's syndrome and achieved beneficial results. Some clinicians have made positive attempts and achieved good results. A retrospective study comparing open thoracotomy or VATS for Boerhaave's syndrome reported that VATS may be used as first choice for Boerhaave's syndrome since it is a safe procedure with a complication rate and results the same as those for open thoracotomy[7]. For some lower esophageal perforation, laparoscopic surgery display unique advantage over open surgery, which shows intraoperative precise positioning and enhanced recovery after surgery. Minimally invasive surgical management of Boerhaave's syndrome is feasible and safe, with outcomes which compare favorably to the published literature[8].

Surgical treatment is well established, but the development of interventional endoscopy has proposed new therapies. Indeed, there are several case reports and publications in the literature regarding the endoscopic treatment of Boerhaave's syndrome. Endoscopy is a proven effective method in the diagnosis of Boerhaave's syndrome, particularly in cases in which the diagnosis is suspected but findings on CT are inconclusive, because endoscopy enables specific lesion characterization. Regarding endoscopic treatment, different therapeutic mechanisms can be proposed: closing with over-the-scope-clips $[9,10]$, endoscopic ligation with snare loops[11], derivation indwelling esophageal stent[12, 13], and placement of open-pore polyurethane foam or luminal sponge[14, 15]. From the perspective of trauma degree, endoscopic management is preserved to treat inoperable patients. In spite of the minimally invasion, endoscopic treatment fail to debride and irrigate the polluted pleural space, which may be potential menace in postoperative rehabilitation. By combined VATS and endoscopy and combing their virtues, we can carry out the share of function of both treatments and achieve advantage complementarity. To the best of our knowledge, there isn't report in the literature that describes application of combined VATS and endoscopy for Boerhaave's syndrome patient. We look forward to application of this surgical management for Boerhaave's syndrome. 
Postoperative management is particularly crucial for Boerhaave's syndrome patient. Anti-infection treatment and nutritional support should be focused in postoperative management[16]. Patients should keep fasting per oral and should be placed a nasogastric tube to clear gastric secretory contents and limit further contamination. Intercostal chest tube would be a good choice for chest drainage no matter in surgical management or conservative treatment. Broad-spectrum antibiotics should be given as soon as possible and last for some time. By strengthening nutritional support both primary and secondary esophageal leaks are being treated with reduced hospital stay and early resumption of oral diet. Repeated and regular contrast study should be utilized to ascertain the progress of the treatment. For optimum outcome for management of esophageal perforations in adults a multidisciplinary approach is needed.

\section{Abbreviations}

VATS: video-assisted thoracoscopic surgery

CT: computed tomography

\section{Declarations}

\section{Acknowledgements}

Not applicable.

\section{Authors' contributions}

WJY and CJJ took care of patients in the operating theater and wrote and revised the paper; WDG took care of patients in the ICU department. WDG and CJJ participated in the data analysis and interpretation and substantively revised the manuscript providing important intellectual content.

All authors (1) have read and approved the manuscript, and (2) have agreed both to be personally accountable for the author's own contributions and to ensure that questions related to the accuracy or integrity of any part of the work, even ones in which the author was not personally involved, are appropriately investigated, resolved, and the resolution documented in the literature.

\section{Funding}

This study did not receive any specific grants from any funding agencies in the public, commercial, and non-profit sectors.

\section{Availability of data and materials}

All data generated or analyzed during this study are included in this published article.

\section{Ethics approval and consent to participate}


Since this is a case report, approval from the local ethical committee is not necessary.

\section{Consent for publication}

The patient signed consent for publication of data (including individual details and images).

\section{Conflicts of Interest:}

The authors have no conflicts of interest to declare.

\section{References}

1. Rokicki, M., W. Rokicki, and M. Rydel, Boerhaave's Syndrome - Over 290 Yrs of Surgical Experiences. Surgical, Endoscopic and Conservative Treatment. Pol Przegl Chir, 2016. 88(6): p. 365-372.

2. Velasco Hernandez, D.N., et al., Boerhaave's syndrome with late presentation. Experience in an Argentine single center: Case series. Ann Med Surg (Lond), 2019. 45: p. 59-61.

3. Shaker, $\mathrm{H}$., et al., The influence of the 'golden 24-h rule' on the prognosis of oesophageal perforation in the modern era. Eur J Cardiothorac Surg, 2010. 38(2): p. 216-22.

4. Vest, M.T. and P. Dross, Boerhaave Syndrome. J Am Osteopath Assoc, 2018. 118(11): p. 764.

5. Muresan, M., et al., Sepsis in Acute Mediastinitis - A Severe Complication after Oesophageal Perforations. A Review of the Literature. J Crit Care Med (Targu Mures), 2019. 5(2): p. 49-55.

6. Han, D., et al., The Role of Operation in the Treatment of Boerhaave's Syndrome. 2018. 2018: p. 8483401.

7. Haveman, J.W., et al., Adequate debridement and drainage of the mediastinum using open thoracotomy or video-assisted thoracoscopic surgery for Boerhaave's syndrome. Surg Endosc, 2011. 25(8): p. 2492-7.

8. Elliott, J.A., et al., Minimally invasive surgical management of spontaneous esophageal perforation (Boerhaave's syndrome). Surg Endosc, 2019.

9. Bona, D., et al., Management of Boerhaave's syndrome with an over-the-scope clip. Eur J Cardiothorac Surg, 2014. 45(4): p. 752-4.

10. Lee, H.L., et al., Efficacy of the Over-the-Scope Clip System for Treatment of Gastrointestinal Fistulas, Leaks, and Perforations: A Korean Multi-Center Study. Clin Endosc, 2018. 51(1): p. 61-65.

11. Kuwabara, J., et al., Successful closure of spontaneous esophageal rupture (Boerhaave's syndrome) by endoscopic ligation with snare loops. Springerplus, 2016. 5(1): p. 921.

12. Y, K., et al., Boerhaave syndrome in an elderly man successfully treated with 3-month indwelling esophageal stent. Radiol Case Rep, 2018. 13(5): p. 1084-1086.

13. Chen, A. and R. Kim, Boerhaave syndrome treated with endoscopic suturing. VideoGIE, 2019. 4(3): p. 118-119. 
14. Loske, G., et al., Endoscopic vacuum therapy in Boerhaave's syndrome with open-pore polyurethane foam and a new open-pore film drainage. Endoscopy, 2015. 47 Suppl 1 UCTN: p. E410-1.

15. Scharl, M., et al., Successful treatment of a proximal esophageal rupture with a luminal sponge. Endoscopy, 2015. 47 Suppl 1 UCTN: p. E293-4.

16. Aloreidi, K., et al., Non-surgical management of Boerhaave's syndrome: a case series study and review of the literature. Endosc Int Open, 2018. 6(1): p. E92-e97.

\section{Figures}
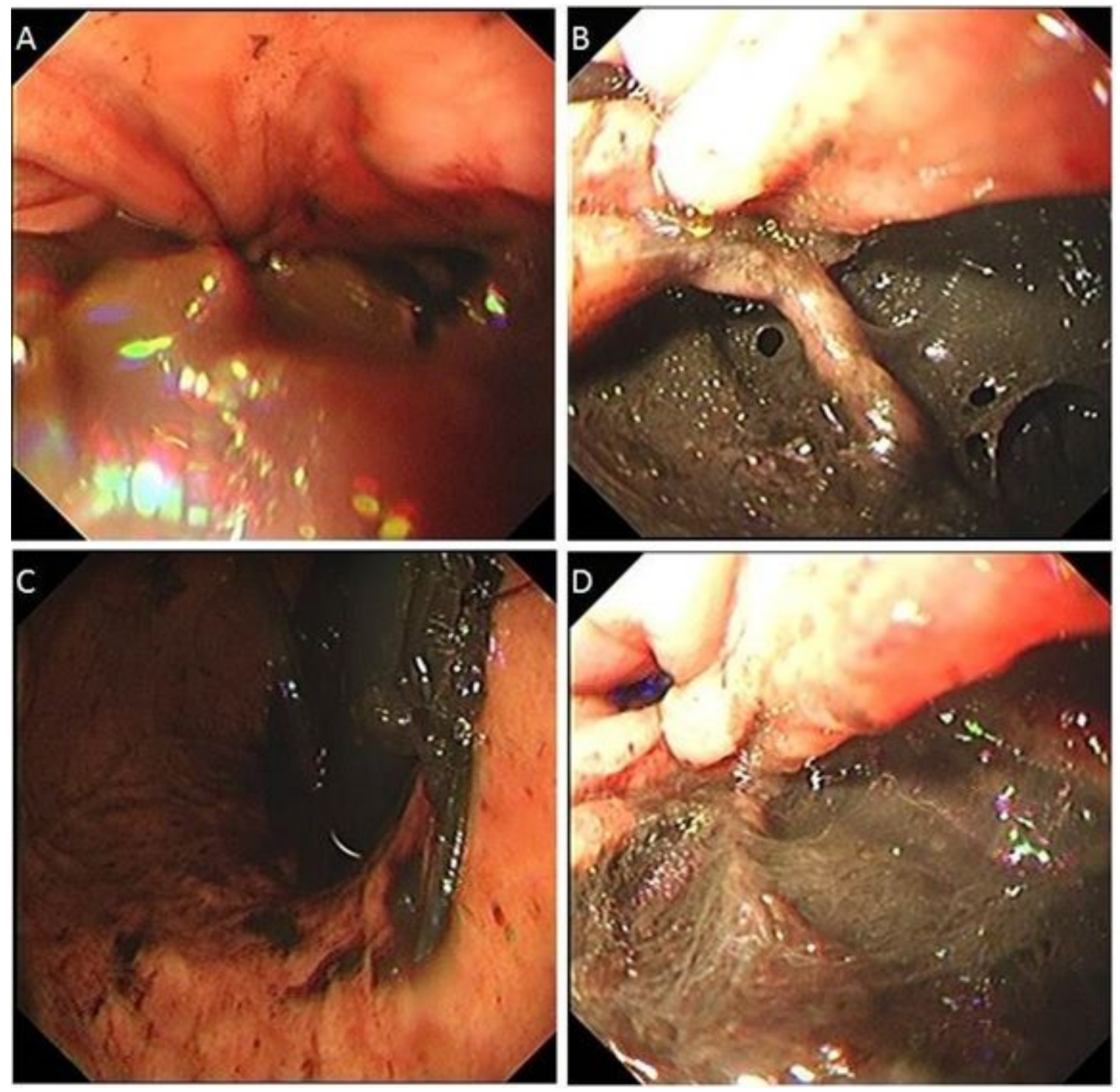

\section{Figure 1}

Upper gastrointestinal endoscopy revealed a full-thickness rupture of the esophageal wall. 


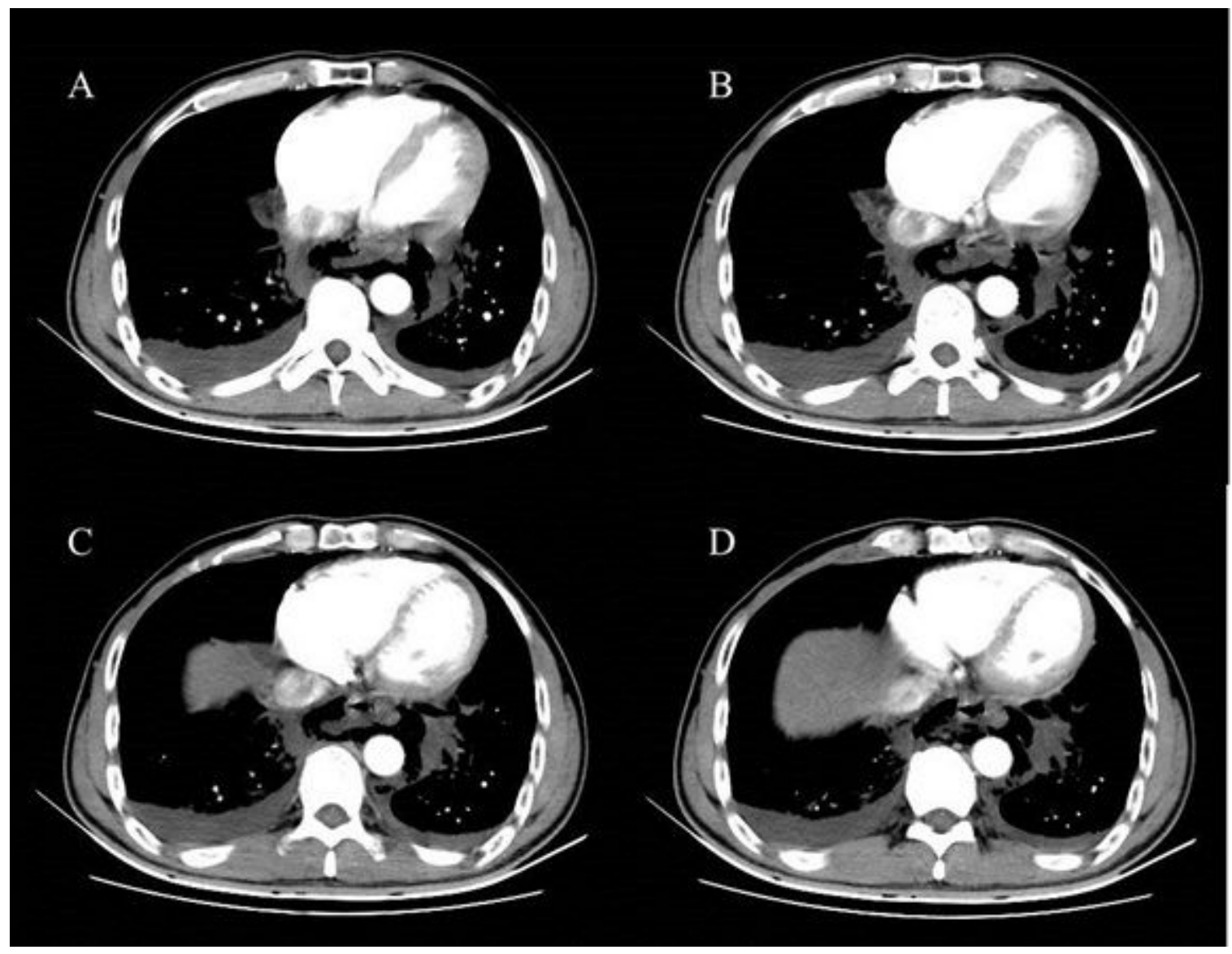

Figure 2

CT showed oesophageal rupture with air leakage into the mediastinum and heterogeneous pleural effusion. 


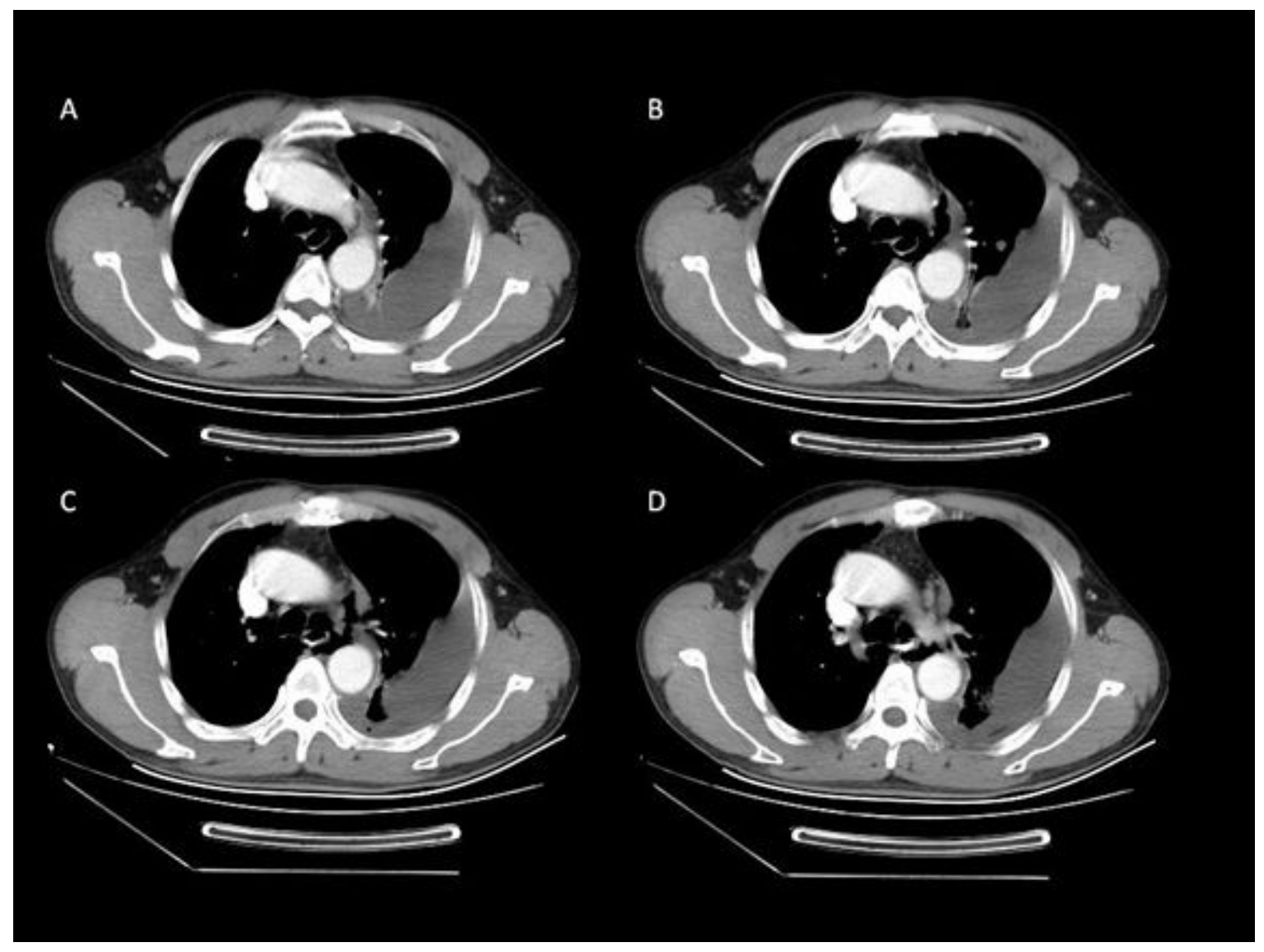

Figure 3

CT showed a tear in the left anterolateral wall of the distal oesophagus, pneumomediastinum and left pleural effusions.
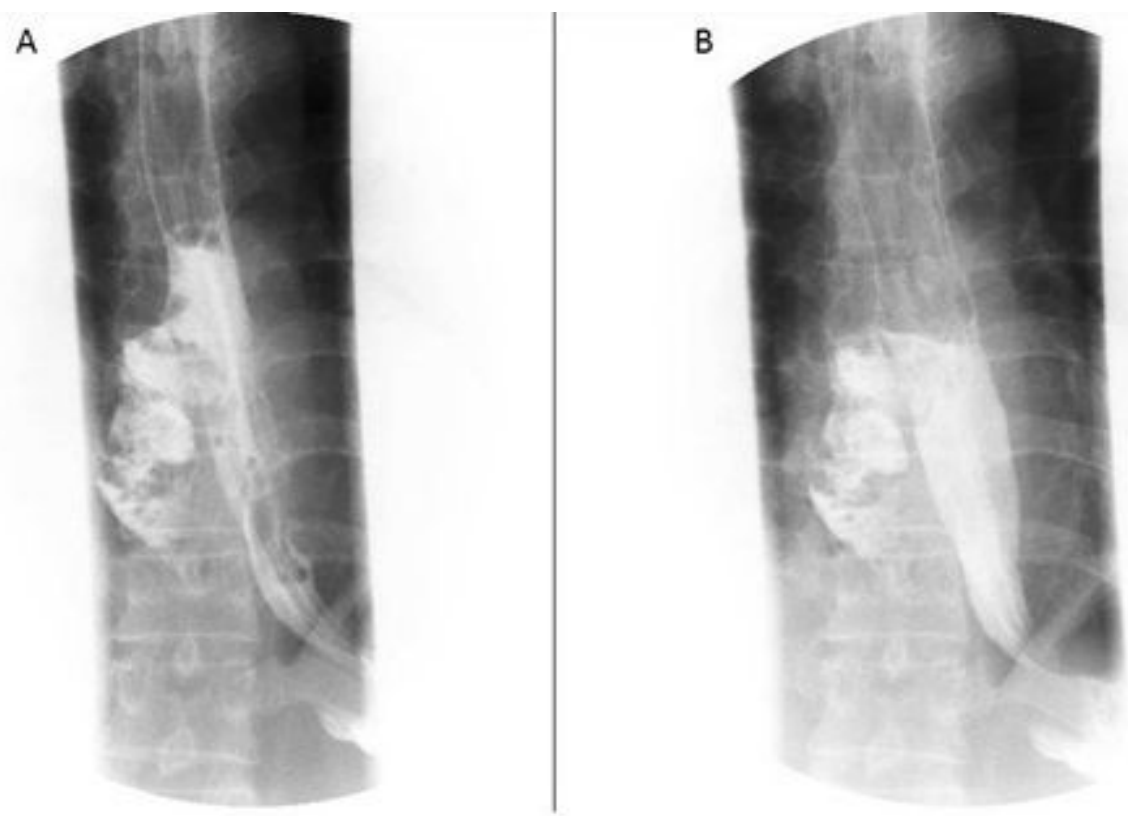

\section{Figure 4}

Esophagus fistula was further confirmed by contrast esophagography. 


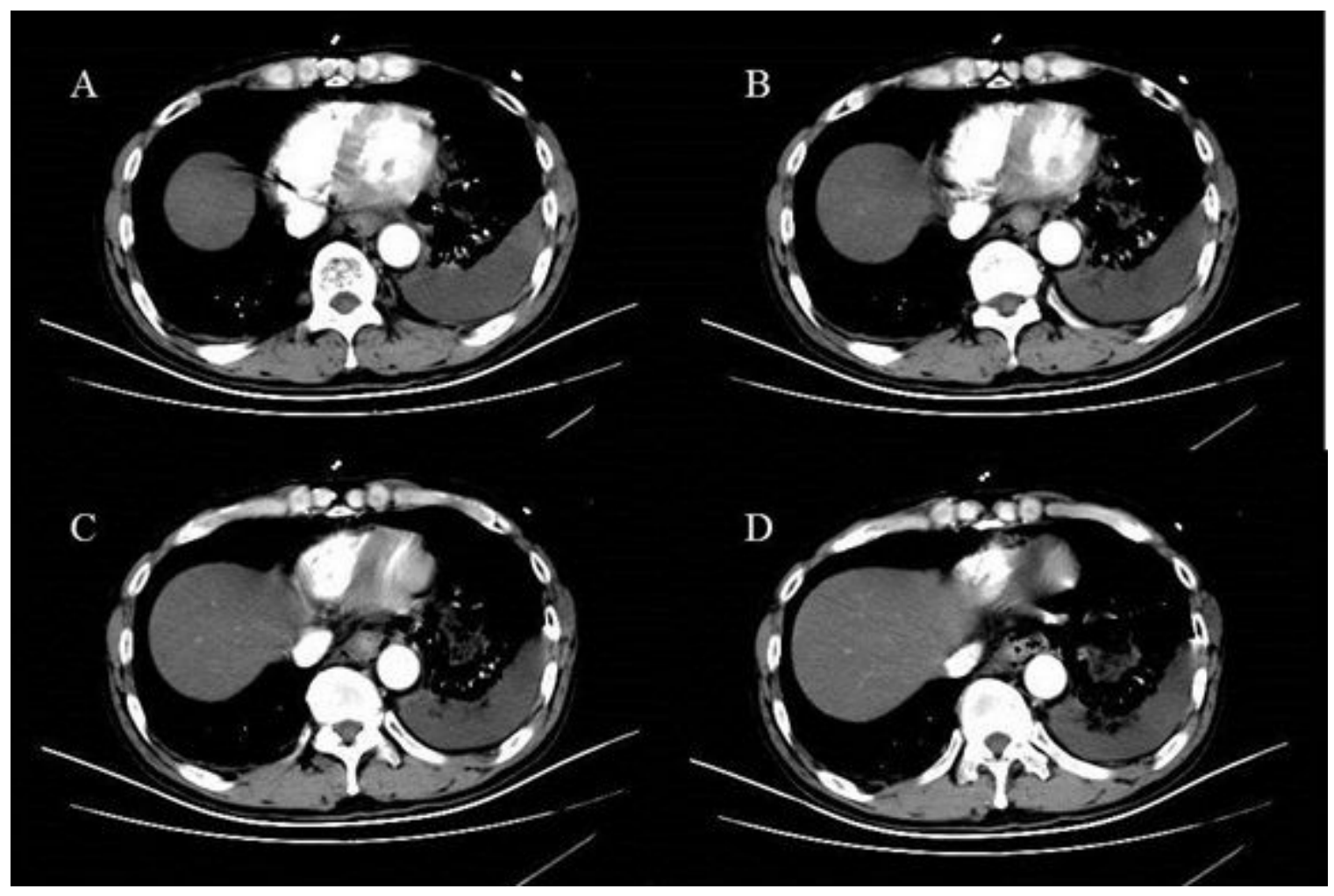

Figure 5

CT showed pneumomediastinum with air tracking laterally towards the left pleural space, and a large left pleural effusion. 